\title{
Regulatory and scientific barriers to the safety evaluation of medical nanotechnologies
}

\section{"Regulatory agencies face significant barriers to the reform of regulatory guidance for nanotoxicological evaluation ... The future of nanotechnology-based medicine will depend on how these challenges are addressed."}

Nanotechnology has permeated several key markets, with its fastest and highest rate of incorporation found in the development of medicinal products, medical device and other healthcare products. This has been achieved without reform of regulatory testing guidelines that were developed before nanotechnologies were even conceptualized.

A number of medicinal products containing nanomaterials have already been authorized both in the EU and the USA. These nanotechnology-based products incorporate nanoscale liposomes (e.g., Caelyx ${ }^{\circledR} /$ Doxil $^{\circledR}$ and Myocet $^{\mathrm{TM}}$ ), lipid micelles (e.g., Estrasorb ${ }^{\circledR}$ ), virosomes (e.g., Epaxal Berna ${ }^{\circledR}$ ) and pegylated proteins (e.g., Neulasta ${ }^{\circledR}$, Pegasys ${ }^{\circledR}$, PegIntron ${ }^{\circledR}$, Macugen ${ }^{\circledR}$ and Somavert $\left.{ }^{\circledR}\right)$. Other products include CALAA-01 with its proprietary siRNApolymer delivery system, the synthetic amino acid polymer in Copaxone ${ }^{\circledR}$, protein conjugates (e.g., Abraxane ${ }^{\circledR}$ ), microemulsions (e.g., of cyclosporine) and nanocrystalline suspensions (e.g., Rapamune ${ }^{\circledR}$, Emend $^{\circledR}$ and Megace ES). Additional organic nanomaterials being investigated for drug delivery include carbon fullerenes and nanotubes, whilst inorganic nanoparticles are already incorporated into a number of in vivo diagnostic products (iron nanoparticles), wound care products (nanosilver) or dental and bone restoratives (calcium nanoparticles and composites) [1].

The fact that novel characteristics of nanomaterials, such as Silcryst ${ }^{\mathrm{TM}}$ and $\mathrm{NanoO}{ }^{\circledR}{ }^{\circledR}$, are conferred by their size, shape, surface and core chemistry have been long since recognized by organizations such as the US and European Patent and Trademark Offices and regulatory agencies such as the European Medicines Agency (EMEA) and the US FDA. These novel characteristics have met important medical needs, such as the ability to deliver therapeutic agents across biological membranes, improve their solubility, reduce their toxicity or allow their localized concentration at tumor sites. However, in spite of a growing body of evidence that many of the desirable properties of nanomaterials can also translate to risks, to date there is no specific guidance pertaining to the safety evaluation of nanotechnology-based products. One such risk is the ability of nanomaterials to cross biological barriers impervious to larger particles. In some cases, this may trigger neurotoxicity [2]. Furthermore, chemical composition does not necessarily correlate with risk. Indeed, last year it was reported that engineered carbon nanotubes, once thought to be no more toxic than graphite, trigger the formation of lesions akin to those seen in asbestosis in mice [3]. In light of such evidence, in April 2009, the EU Parliament called the Commission to task over its decision to allow single registration of nano and bulk equivalent materials under the EU Chemicals Policy after having already come to the conclusion that existing test methods are not adequate for assessing the risks associated with nanomaterials in foods and having earlier this year called upon the Commission to compile a database of nanomaterials used in the EU by 2011 and to review legislative measures to ensure the safety of nanomaterials for all uses.

\section{“...in spite of a growing body of evidence that many of the desirable properties of nanomaterials can also translate to risks, to date there is no specific guidance pertaining to the safety evaluation of nanotechnology-based products."}

This begs the question as to why, more than a decade after the first nanotechnologies infiltrated the medical care market, there are no specific guidelines for the evaluation of nanotechnology-based medical products.

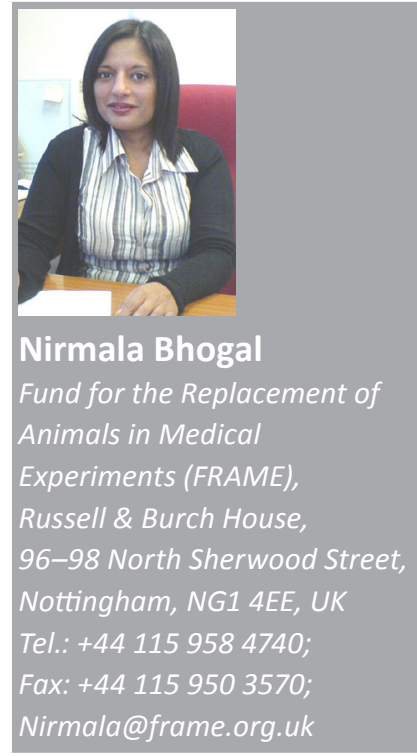

future medicti $^{\text {pos }}$ fsg 
Despite the variety of definitions put forward, it is immediately clear that nanotechnology is a term that broadly encompasses any technology that involves the use of materials of a nanoscale, but which does not distinguish between inert and reactive components or between medicinal products and devices. In 2006, the EMEA published a reflection paper, within which it was acknowledged that nanotechnologies blur the demarcation between medicinal products and medical devices, such that applicants were advised to seek early guidance from the EMEA as to appropriate testing under the existing regulatory framework [101]. This document more importantly indicated that, whilst the existing framework can accommodate evaluation of existing nanotechnology-based products, revision of the guidelines would almost certainly be necessary in the longer term. This view is broadly consistent with opinions expressed in 2007 by the European Group on Ethics in Science and New Technologies to the Commission [102] and in a report by the Commission's New and Emerging Technologies Working Group [103], that contained provisos that current procedures may require modification to take into account the novel properties and uncertainties pertaining to nanomaterials.

\section{"This begs the question as to why, more than a decade after the first nanotechnologies infiltrated the medical care market, there are no specific guidelines for the evaluation of nanotechnology-based medical products."}

In the USA, the Environmental Protection Agency (EPA) is of the opinion that nanomaterials should be regulated in the same way as bulk materials with the same chemical structure, broadly reflecting the EU Commission's, EMEA's and FDA's standpoints. In 2006, the FDA initiated a public information gathering exercise to determine whether nanotechnologies pose novel risks. The exercise was repeated 2 years later. In March 2009, the FDA, although insistent that the present regulatory scheme is adequate for analyzing nanotechnology products, had announced a collaboration with the Houston-based Alliance for NanoHealth in order to expedite the development of safe and effective medical products and expand knowledge about how nanoparticles behave [104].

The main problem faced by the FDA and the EMEA is the fact that there is simply not enough information available about the interactions between nanomaterials and biological systems. There is also insufficient knowledge about how the characterization of nanomaterials with regards to their influence on biological activity, human and environmental biopersistence and short- and long-term toxicity can be undertaken. Many of these problems stem from the lack of sufficiently sensitive and reliable methods to reproducibly measure and characterize potentially heterogeneous subpopulations of nanomaterials. Still further problems arise because most of the existing methodologies, although they may have been validated for or have an established use in the assessment of small chemical substances, have not been assessed for relevance to the evaluation of nanomaterials. Furthermore, limited human exposure to engineered nanoparticles does not lend itself to epidemiological studies. In the absence of such information, a formal evaluation of paradigms developed specifically for the assessment of chemicals in terms of their relevance to nanomaterial safety assessment is problematic.

\section{"The evaluation of completed products, even those that contain approved therapeutic agents, is perhaps the way forward."}

Whilst compiling data about the safety and the future uses of nanotechnologies is a reasonable starting point towards addressing these problems, a major stumbling block is likely to be identifying the key physiochemical determinants of nanomaterials that will enable their categorization based on the likely regulatory testing needs. Assuming categorization is possible, the use of in silico prediction models of activity or toxicity may facilitate the identification and development of tests relevant to the evaluation of nanomaterials. However, recent discussions with regards to the implementation of the EU Chemicals Policy suggest that in silico prediction models for the risk assessment of nanomaterials will depend on the availability of information for a wide range of nanomaterials. Even so, subtle changes in chemistry, shape, size and surface functionality and propensity to aggregate and disaggregate will introduce variables that may not be able to be readily accommodated within existing mathematical models $[4,5]$.

Another pertinent issue is whether testing of the nanomaterial component of a product gives a reliable indication of in-product in vivo interactions. The evaluation of completed products, even those that contain approved therapeutic agents, is perhaps the way forward. However, 
this may create an additional burden for the identification of relevant in vivo preclinical models, since species differences in nanomaterial biodistribution and handling are already suspected to exist. This, in turn, may create the need for supplementary studies in additional animals, with its commensurate animal welfare, time and economic costs. Species differences in Peyer's patch density, distribution and gut $\mathrm{pH}$ may, for instance, have a significant bearing on oral biovailability of encapsulated therapeutics [6]. Likewise, although species differences in the route and rate of small chemical uptake through the skin has been attributed to follicular diameter or density at different body sites [7] and this may be a possible route of dermal uptake for nanomaterials, the relevance of these data for small chemicals is unclear.

Another confounding factor to the development of regulatory guidelines specifically for nanomaterials is the discordance between the available data regarding the safety and biodistribution of nanomaterials. These problems are partly attributed to the variety of animal models. Cross-study comparison is also complicated because current analytical methods are limited in their ability to accurately determine nanomaterial heterogeneity resulting from poor size or functionality-based separation of nanoparticles or from particle aggregation, modification or degradation. In other cases, although similar size nanoparticles have been studied in the same species, the particle composition differs. This may explain why it is not clear, for instance, whether nanoparticles smaller than $10 \mathrm{~nm}$ are deposited in the peripheral lung or exhaled again $[8,9]$.
In vitro cell culture and tissue equivalent models, based on the use of well-characterized cells and cell lines, play a vital role in guiding nanotechnological development. Perhaps some of the best characterized human tissue models are those that are now routinely used to assess the dermal penetration and toxicity of cosmetic products. Whether these are suitable for the evaluation of transdermal penetration and toxicity of nanomaterials, however, remains to be established, as is also the case for most animal tests.

\section{Conclusion}

Regulatory agencies face significant barriers to the reform of regulatory guidance for nanotoxicological evaluation. These problems stem from the limited availability of information regarding the relevance of physicochemical properties of nanomaterials to the risks they pose to human health and the environment. Furthermore, preclinical screens and animal models have not been formally validated for the assessment of nanomaterials. The future of nanotechnology-based medicine will depend on how these challenges are addressed.

\section{Financial \& competing interests disclosure}

The author has no relevant affiliations or financial involvement with any organization or entity with a financial interest in or financial conflict with the subject matter or materials discussed in the manuscript. This includes employment, consultancies, honoraria, stock ownership or options, expert testimony, grants or patents received or pending, or royalties.

No writing assistance was utilized in the production of this manuscript.

\section{Bibliography}

1 Wagner V, Dullart A, Bock A-K, Zweck A: The emerging nanomedicine landscape. Nat. Biotechnol. 24(10), 1211-1217 (2006).

2 Long TC, Saleh N, Tilton RD, Lowry GV, Veronesi B: Titanium dioxide (P25) produces reactive oxygen species in immortalized brain microglia (BV2): implications for nanoparticle neurotoxicity. Environ. Sci. Technol. 40, 4346-4352 (2006).

3 Poland CA, Duffin R, Kinloch I et al.: Carbon nanotubes introduced into the abdominal cavity of mice show asbestos-like pathogenicity in a pilot study. Nat. Nanotechnol. 3, 423-428 (2008).

4 Oberdörster G, Maynard A, Donaldson K et al: : Principles of characterizing the potential health effects from exposure to nanomaterial: elements of a screening strategy. A report from
ILSI Research foundation/Risk science instate Nanomaterial Toxicity screening Working Groups. Particle Fibre Toxicol. 2, 1-36 (2005).

5 Labhesetwar V, Song C, Humphrey W, Shebucki R, Levy RJ: Arterial uptake of biodegradable nanoparticles: effects of surface modifications. J. Pharm. Sci. 87, 1229-1234 (2000).

Harashima H, Komatsu S, Kojima S et al.: Species difference in the disposition of liposomes among mice, rats, and rabbits: allometric relationship and species dependent hepatic uptake mechanism. Pharmacol. Res. 13, 1049-1054 (1996).

7 Scott RC, Corrigan MA, Smith F, Mason H: The influence of skin structure on permeability: an intersite and interspecies comparison with hydrophilic penetrants. J. Invest. Dermatol. 96, 921-925 (1991).
8 Oberdorster G, Sharp Z, Atudorei V et al:: Extrapulmonary translocation of ultrafine carbon particles following whole-body inhalation exposure of rats. J. Toxicol. Environ. Health A 65, 1531-1543 (2002).

9 Takenaka S, Karg E, Kreyling WG et al:: Distribution pattern of inhaled ultrafine gold particles in the rat lung. Inhal. Toxicol. 18, 733-740 (2006).

\section{Websites}

101 Committee for Medicinal Products for Human Use - CHMP (2006). Reflection paper on Nanotechnology-Based Medicinal Products for Human Use, EMEA/ CHMP/79769/2006. www.emea.europa.eu/pdfs/human/ genetherapy/7976906en.pdf (Accessed 1 May 2009) 
102 EGE (2007). Opinion 21 - On the ethical aspects of nanomedicine.

http://ec.europa.eu/european_group_ethics/ publications/docs/final_publication_\%20 op21_en.pdf

(Accessed 1 May 2009)

103 N\&ET Working Group (2007). Report on nanotechnology to the medical devices expert group findings and recommendations. http://ec.europa.eu/enterprise/medical_ devices/net/entr-2007-net-wg-report-nanofinal.pdf

(Accessed 1 May 2009)

104 Memorandum of understanding between the United States Food and Drug Administration, Baylor College of Medicine, The University of Texas M.D. Anderson Cancer Center, Rice University, University of Houston,
The University of Texas Health Science Center at Houston, Texas A\&M Health Science Center, The University of Texas Medical Branch at Galveston, The Methodist Hospital Research Institute for the FDAANH Nanotechnology Initiative. www.fda.gov/oc/mous/ academic/225-207-8006.html (Accessed 1 May 2009) 